\title{
Prematuridade no Brasil entre 2012 e 2019: dados do Sistema de Informações sobre Nascidos Vivos ${ }^{\star}$
}

\author{
Katrini Guidolini Martinelli ${ }^{\star \star}$ \\ Barbara Almeida Soares Dias ${ }^{\star \star \star}$ \\ Marcelle Lemos Leal ${ }^{\star \star \star \star}$ \\ Lorrayne Belotti \\ Érica Marvila Garcia \\ Edson Theodoro dos Santos Neto ${ }^{\star \star \star \star \star \star \star}$
}

O objetivo deste estudo é analisar a tendência de prematuridade no Brasil, entre 2012 e 2019, segundo características sociodemográficas, do pré-natal e parto. Trata-se de um estudo ecológico de série temporal, em que foram calculadas as proporções de prematuridade segundo as regiões do Brasil, idade materna, escolaridade materna, raça/cor, número de consultas pré-natal e tipo de parto, a partir dos dados do Sistema de Informações sobre Nascidos Vivos (Sinasc). Sequencialmente, foram aplicados modelos de regressão polinomial para análise de tendência temporal. De 2012 a 2019, a proporção de prematuridade no Brasil apresentou tendência decrescente, variando de $10,87 \%$ a $9,95 \%$, com a menor proporção em 2015 , que foi de $9,77 \%$. As mulheres na faixa etária de 45 anos ou mais e com 4 a 6 consultas de pré-natal apresentaram as maiores proporções de prematuridade para o período (14,88\% a 17,92\%) e com tendência crescente. Já as mulheres analfabetas e indígenas mostraram tendência decrescente para o período, apesar de terem as maiores proporções de prematuridade (15,75\% a 11,74\%). Há uma tendência decrescente de prematuridade no Brasil, especialmente em mulheres mais vulneráveis, entretanto, os serviços de saúde precisam melhorar o atendimento das mulheres com idade materna avançada e atrair aquelas com poucas consultas de pré-natal.

Palavras-chave: Recém-nascido prematuro. Nascimento prematuro. Sistemas de informação. Planejamento em saúde.

\footnotetext{
* 0 presente artigo é um produto do projeto de pós-doutorado de Katrini Guidolini Martinelli, financiado pela Fundação de Amparo à Pesquisa e Inovação do Espírito Santo (Fapes). Edital Profix Fapes/Capes n. 10/2018, processo n. 83552936.

** Universidade Federal do Espírito Santo (Ufes), Vitória-ES, Brasil (katrigm@gmail.com; https://orcid.org/0000-00030894-3241).

*** Escola Nacional de Saúde Pública Sérgio Arouca (Ensp), Fundação Oswaldo Cruz (Fiocruz), Rio de Janeiro-RJ, Brasil (barbaraalmeidasd@gmail.com; https://orcid.org/0000-0001-8656-1391).

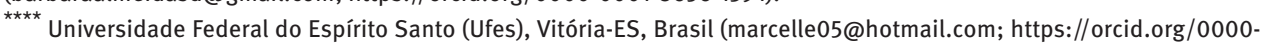
0001-7878-6654).

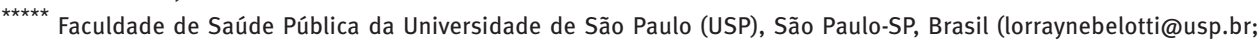
https://orcid.org/0000-0001-6111-8908).

${ }^{\star \star \star \star \star \star}$ Faculdade de Saúde Pública da Universidade de São Paulo (USP), São Paulo-SP, Brasil (ericamarvila@usp.br; https:// orcid.org/0000-0001-9477-2383).

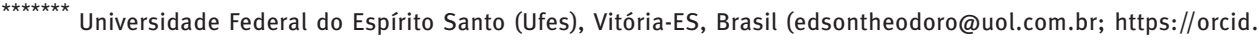
org/0000-0002-7351-7719).
} 


\section{Introdução}

Prematuridade é todo nascimento ocorrido antes de 37 semanas completas de gestação, podendo ser classificada, segundo a idade gestacional (IG), em prematuridade extrema (de 22 a menos de 28 semanas), prematuridade severa (de 28 a menos de 32 semanas) e prematuridade moderada a tardia (de 32 a menos de 37 semanas) (HOWSON et al., 2012). Diferentes fatores influenciam a ocorrência da prematuridade, tais como genéticos, sociodemográficos (MARTIN, 2017), ambientais (HUANG et al., 2018) e principalmente aqueles relacionados à gestação (ABDEL RAZEQ; KHADER; BATIEHA, 2017).

De acordo com a Organização Mundial da Saúde (OMS), a proporção de prematuridade vem aumentando nas últimas décadas (HOWSON et al., 2012). Em 2014 ocorreram 14,8 milhões de nascimentos prematuros no mundo, o que representou $10,6 \%$ de todos os nascimentos (CHAWANPAIBOON et al., 2019). No Brasil, houve uma tendência crescente na proporção de prematuros entre 1994 e 2005 (SILVEIRA et al., 2009), a qual ficou em torno de $11 \%$ no período de 2005 a 2011 (MATIJASEVICH et al., 2013), sendo considerada elevada quando comparada à dos países europeus, que registraram $8,7 \%$ de prematuridade (CHAWANPAIBOON et al., 2019).

A prematuridade está intimamente relacionada à morbimortalidade infantil, sendo uma das principais causas de morte no período neonatal (GONZAGA et al., 2016). Além disso, o manejo de um nascido vivo prematuro gera custos elevados para o setor da saúde (SILVEIRA et al., 2009). Por isso é essencial a avaliação do desempenho do sistema de saúde por meio do monitoramento das tendências e mudanças dos indicadores de saúde, incluindo a prematuridade (SILVEIRA et al., 2009).

No Brasil, estudos com dados primários mostravam uma proporção de prematuridade superior à encontrada nos dados oficiais do Sistema de Informações sobre Nascidos Vivos (Sinasc) até 2010, pois a idade gestacional (IG) era classificada de modo agrupado e sem especificar o seu método de estimação (MATIJASEVICH et al., 2013). Somente a partir de 2011 a IG passou a ser registrada de forma desagregada em semanas gestacionais, sendo estimada preferencialmente pela data da última menstruação (DUM). Contudo, quando desconhecida a DUM, outros métodos de estimação, como exame físico ou ultrassonografia, devem ser utilizados para estimar a IG (BRASIL, 2011).

Tendo em vista a subestimação da proporção de prematuridade no Brasil com dados oficiais até 2011 (MATIJASEVICH et al., 2013), a associação entre prematuridade e fatores sociodemográficos e relacionados à assistência pré-natal e parto (BALBI; CARVALHAES; PARADA, 2016) e a importância de monitorar a tendência da prematuridade, este estudo tem como objetivo analisar a tendência de prematuridade no Brasil entre 2012 e 2019, segundo características sociodemográficas, do pré-natal e parto. 


\section{Métodos}

Trata-se de estudo ecológico de série temporal, baseado em dados secundários de nascidos vivos no Brasil no período de 2012 a 2019. Utilizaram-se registros do Sistema de Informações sobre Nascidos Vivos (Sinasc) extraídos do sítio eletrônico do Departamento de Informática do Sistema Único de Saúde (Datasus) do Ministério da Saúde (http://tabnet. datasus.gov.br/cgi/deftohtm.exe?sinasc/cnv/nvuf.def.). A coleta de dados ocorreu em fevereiro de 2021.

Para este estudo foram selecionadas as seguintes variáveis:

- número de nascidos vivos - consideraram-se apenas as gestações com idade gestacional maior do que 22 semanas e peso ao nascer superior a 1.500 gramas, para não haver erro na classificação com natimortos, já que em países de média e baixa renda há, com frequência, erro na classificação desses recém-nascidos (CHAWANPAIBOON et al., 2019);

- idade gestacional em semanas - prematuro extremo (22-27 semanas gestacionais), prematuro severo (28-31 semanas gestacionais), prematuro moderado a tardio (32-37 semanas gestacionais) e semana gestacional ignorada;

- macrorregião de residência da mãe - Norte, Nordeste, Centro-Oeste, Sudeste e Sul;

- idade materna - 10-14 anos, 15-19 anos, 20-34 anos, 35-39 anos, 40-44 anos e 45 anos ou mais;

- escolaridade materna - analfabeta, 1-7 anos de estudo, 8-11 anos e 12 anos ou mais;

- raça/cor materna - branca, preta, amarela, parda e indígena;

- número de consultas pré-natal - nenhuma, 1-3 consultas, 4-6 consultas e 7 consultas ou mais;

- tipo de parto - vaginal e cesáreo.

Os cálculos das proporções de prematuridade foram realizados segundo as subcategorias de idade gestacional. Dividiu-se, então, o número de prematuros de cada subcategoria pelo total de nascidos vivos e multiplicou-se por 100. Para a análise da prematuridade total (22-37 semanas gestacionais), segundo idade materna, escolaridade materna, tipo de parto, número de consultas pré-natal e raça/cor, dividiu-se o número de prematuros para cada categoria da variável pelo total de nascidos vivos da categoria.

As séries resultantes desta análise tiveram suas tendências estimadas pelo método de regressão polinomial. Assim, foram realizados diagramas de dispersão entre a proporção de prematuridade e os anos de estudo com o intuito de visualizar a função que poderia expressar a relação entre eles. A partir da relação funcional observada, foram estimados modelos de regressão polinomial.

No processo de modelagem, os indicadores referentes à proporção de prematuridade (segundo cada subcategoria) foram considerados como variável dependente (Y) e os anos 
de estudo como variável independente (X). Dessa forma, foi realizada a transformação da variável "ano" na variável ano-centralizada, ou seja, a variável "ano" menos o ponto médio do período de estudo. Essa etapa foi importante, pois em modelos de regressão polinomial os termos da equação são com frequência altamente correlacionados e, portanto, expressar a variável independente como um desvio de sua média reduz substancialmente a autocorrelação entre eles (LATORRE; CARDOSO, 2001). Os coeficientes de determinação ( $R^{2}$ e $R^{2}$ ajustado) foram utilizados como medida de precisão do modelo.

Para verificar se os dados apresentavam distribuição normal, foi realizado o teste de Kolmogorov-Smirnov. Todas as séries apresentaram distribuição normal e, portanto, aceitou-se a hipótese nula de que os dados seguem uma distribuição normal ( $p>0,05)$. Por fim, foi feita a análise dos resíduos, confirmando a suposição de homocedasticidade do modelo.

Inicialmente testou-se o modelo de regressão linear simples $\left(Y=\beta_{0}+\beta_{1} X\right)$ e, em seguida, foram testados os modelos de segundo grau $\left(Y=\beta_{0}+\beta_{1} X+\beta_{2} X_{2}\right)$ e terceiro grau $\left(Y=\beta_{0}+\beta_{1} X+\beta_{2} X_{2}+\beta_{3} X_{3}\right)$. Os testes foram realizados sequencialmente. Quando dois modelos foram semelhantes do ponto de vista estatístico, optou-se pelo mais simples, ou seja, o de menor ordem. Considerou-se tendência significativa aquela cujo modelo estimado obteve p<0,05 (LATORRE; CARDOSO, 2001).

Os cálculos das proporções de prematuridade e os gráficos de séries históricas foram elaborados no Microsoft Office Excel (versão 14.0 para Windows 2016) e as análises de tendências foram realizadas no programa STATA, versão 14.1.

Por utilizar exclusivamente dados secundários e disponibilizados publicamente, foi dispensada a apreciação por Comitê de Ética em Pesquisa com Seres Humanos, de acordo com as Resoluções 466/2012, 510/16 e 580/18 do Conselho Nacional de Saúde do Brasil.

\section{Resultados}

Entre 2012 e 2019, foram registrados 23.059 .611 nascidos vivos no Sinasc, dos quais $20.574(0,09 \%)$ eram prematuros extremos, $122.132(0,53 \%)$ prematuros severos e $2.188 .723(9,49 \%)$ prematuros moderados ou tardios. A proporção de prematuridade total no Brasil variou de $10,87 \%$ a 9,95\% no período estudado, com a menor em 2015 (9,77\%). A proporção de semana gestacional ignorada variou de 4,81\% a 1,27\% (Gráfico 1), com tendência decrescente para o período (Tabela 1). 
GRÁFICO 1

Proporção de prematuridade, segundo subcategorias de idade gestacional

Brasil - 2012-2019

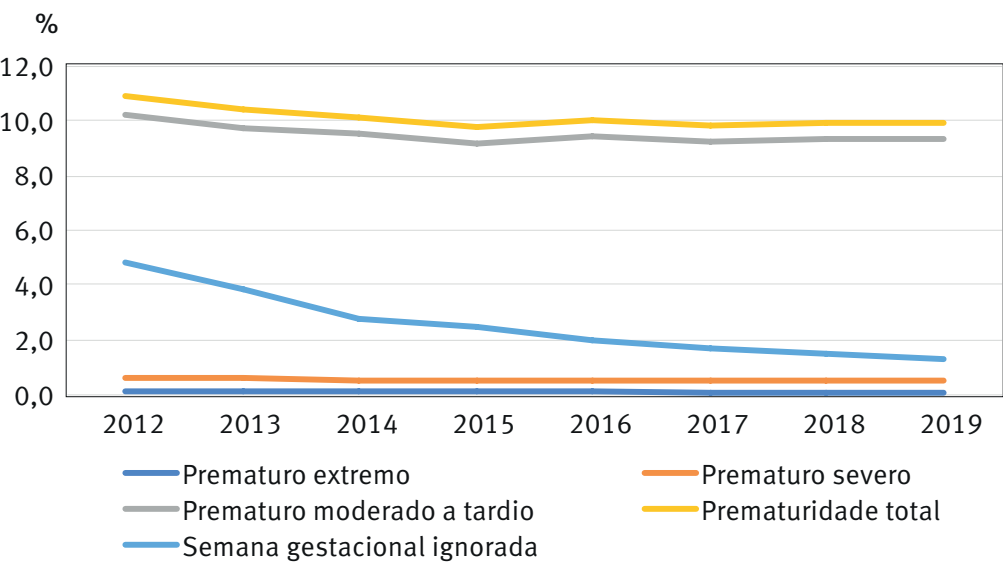

Fonte: Sistema de Informações sobre Nascidos Vivos (Sinasc). Elaboração dos autores.

TABELA 1

Análise de tendência temporal da proporção de prematuridade, segundo subcategorias de idade gestacional e macrorregiões

Brasil - 2012-2019

\begin{tabular}{|c|c|c|c|c|c|c|c|c|}
\hline $\begin{array}{l}\text { Idade gestacional } \\
\text { e macrorregiões }\end{array}$ & Modelo & $\mathrm{R}$ & $\mathrm{R}^{2}$ & $\beta_{0}$ & $\beta_{1}$ & $\beta_{2}$ & $\beta_{3}$ & p-valor \\
\hline \multicolumn{9}{|l|}{$\begin{array}{l}22 \text { a } 27 \text { semanas } \\
\text { (prematuro extremo) }\end{array}$} \\
\hline Norte & Linear & 0,904 & 0,880 & 0,140 & $-0,005$ & - & - & 0,004 \\
\hline Nordeste & Linear & 0,979 & 0,973 & 0,133 & $-0,007$ & - & - & $<0,001$ \\
\hline \multirow[t]{3}{*}{ Centro-Oeste (1) } & Linear & 0,041 & $-0,199$ & 0,064 & $-0,001$ & - & - & 0,701 \\
\hline & Parábola & 0,696 & 0,493 & 0,594 & $-0,001$ & 0,002 & - & 0,168 \\
\hline & Terceiro grau & 0,964 & 0,911 & 0,594 & $-0,005$ & 0,002 & 0,001 & 0,053 \\
\hline Sudeste & Linear & 0,976 & 0,969 & 0,062 & $-0,004$ & - & - & $<0,001$ \\
\hline \multirow[t]{3}{*}{ Sul } & Linear & 0,428 & 0,285 & 0,045 & $-0,002$ & - & - & 0,159 \\
\hline & Parábola & 0,786 & 0,643 & 0,042 & $-0,002$ & 0,001 & - & 0,099 \\
\hline & Terceiro grau & 0,971 & 0,927 & 0,042 & 0,001 & 0,001 & $-0,001$ & 0,044 \\
\hline Brasil & Linear & 0,983 & 0,979 & 0,089 & $-0,004$ & - & - & $<0,001$ \\
\hline \multicolumn{9}{|l|}{$\begin{array}{l}28 \text { a } 31 \text { semanas } \\
\text { (prematuro severo) }\end{array}$} \\
\hline Norte & Linear & 0,686 & 0,607 & 0,693 & $-0,011$ & - & - & 0,042 \\
\hline Nordeste & Linear & 0,990 & 0,988 & 0,612 & $-0,017$ & - & - & $<0,001$ \\
\hline \multirow[t]{2}{*}{ Centro-Oeste } & Linear & 0,267 & 0,084 & 0,467 & 0,001 & - & - & 0,293 \\
\hline & Parábola & 0,958 & 0,930 & 0,464 & 0,001 & 0,001 & - & 0,001 \\
\hline Sudeste & Linear & 0,827 & 0,783 & 0,463 & $-0,013$ & - & - & 0,012 \\
\hline Sul & Linear & 0,691 & 0,614 & 0,436 & $-0,008$ & - & - & 0,040 \\
\hline Brasil & Linear & 0,907 & 0,883 & 0,526 & $-0,012$ & - & - & 0,003 \\
\hline
\end{tabular}


(Continuação)

\begin{tabular}{|c|c|c|c|c|c|c|c|c|}
\hline $\begin{array}{l}\text { Idade gestacional } \\
\text { e macrorregiões }\end{array}$ & Modelo & $\mathrm{R}$ & $\mathrm{R}^{2}$ & $\beta_{0}$ & $\beta_{1}$ & $\beta_{2}$ & $\beta_{3}$ & p-valor \\
\hline \multicolumn{9}{|c|}{$\begin{array}{l}32 \text { a } 37 \text { semanas } \\
\text { (prematuro moderado } \\
\text { a tardio) }\end{array}$} \\
\hline \multirow[t]{2}{*}{ Norte } & Linear & 0,586 & 0,482 & 10,068 & $-0,104$ & - & - & 0,076 \\
\hline & Parábola & 0,991 & 0,985 & 9,896 & $-0,104$ & 0,059 & - & $<0,001$ \\
\hline Nordeste & Linear & 0,917 & 0,896 & 9,346 & $-0,076$ & - & - & 0,003 \\
\hline \multirow[t]{2}{*}{ Centro-Oeste } & Linear & 0,574 & 0,467 & 9,273 & $-0,057$ & - & - & 0,081 \\
\hline & Parábola & 0,979 & 0,964 & 9,178 & $-0,057$ & 0,033 & - & 0,003 \\
\hline \multirow[t]{2}{*}{ Sudeste } & Linear & 0,638 & 0,548 & 9,372 & $-0,127$ & - & - & 0,057 \\
\hline & Parábola & 0,976 & 0,960 & 9,188 & $-0,127$ & 0,063 & - & 0,004 \\
\hline \multirow[t]{3}{*}{ Sul (1) } & Linear & 0,116 & $-0,105$ & 9,328 & $-0,029$ & - & - & 0,509 \\
\hline & Parábola & 0,730 & 0,549 & 9,195 & $-0,029$ & 0,045 & - & 0,140 \\
\hline & Terceiro grau & 0,940 & 0,851 & 9,195 & 0,073 & 0,045 & $-0,020$ & 0,088 \\
\hline Brasil & Linear & 0,687 & 0,608 & 9,425 & $-0,091$ & - & - & 0,041 \\
\hline Sem. gest. ignorada & Linear & 0,951 & 0,938 & 2,427 & $-0,458$ & - & - & 0,001 \\
\hline
\end{tabular}

Fonte: Sistema de Informações sobre Nascidos Vivos (Sinasc). Elaboração dos autores.

(1) Estacionária.

As proporções de prematuridade extrema e severa mostraram-se baixas durante todo o período estudado, entretanto, houve variação entre as regiões do Brasil, com os maiores percentuais no Norte e Nordeste. Observou-se tendência decrescente, para o período, no Norte, Nordeste e Sudeste, assim como para o Brasil. A prematuridade extrema na região Sul mostrou comportamento decrescente de 2012 a 2015, crescente de 2016 a 2017 e decrescente a partir de 2018, enquanto a prematuridade severa reduziu-se em todo 0 período. Já o Centro-Oeste registrou tendência decrescente de 2012 a 2015 e crescente a partir de 2016 para prematuridade severa (Tabela 1).

O Nordeste e o Brasil apresentaram movimento decrescente nas proporções de prematuridade moderada e tardia para o período analisado. Nas regiões Norte e Sudeste as tendências foram decrescentes de 2012 a 2015 e crescentes a partir de 2016. Para o Centro-Oeste, observaram-se redução de 2012 a 2017 e aumento a partir de 2018 (Tabela 1).

A proporção de prematuridade total foi maior entre mães nas faixas etárias de 10 a 14 anos e de 45 anos ou mais, analfabetas ou com 1 a 7 anos de estudo, indígenas e pretas, com 1 a 6 consultas de pré-natal. Quanto ao tipo de parto, até 2016, a maior proporção de prematuros ocorreu entre os partos vaginais e, posteriormente, passou a ser superior entre os partos cesáreos (Gráfico 2). 
GRÁFICO 2

Proporção de prematuridade, segundo idade da mãe, escolaridade, raça/cor, número de consultas pré-natal e tipo de parto

Brasil - 2012-2019

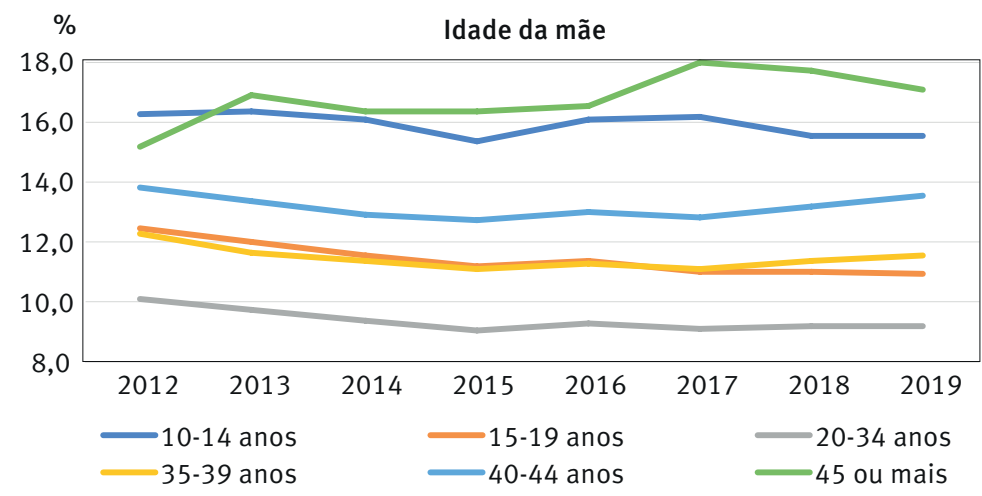

$\% \quad$ Escolaridade
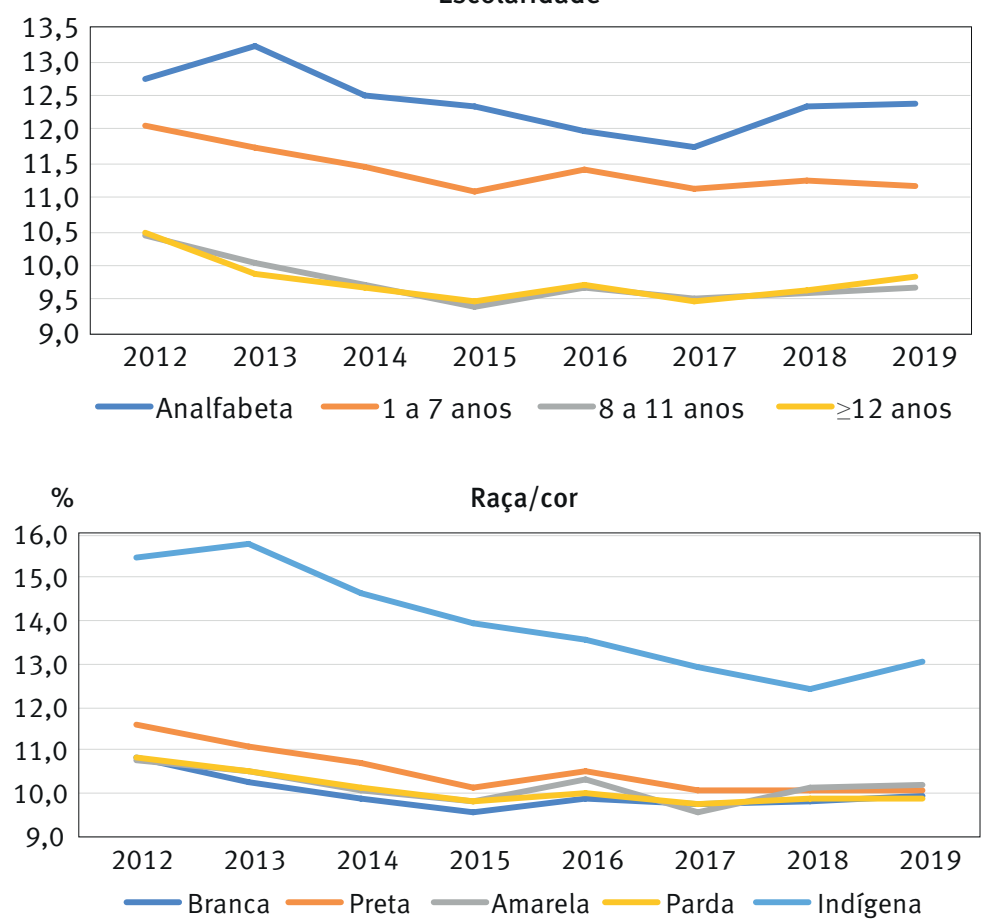

(Continua) 
(Continuação)
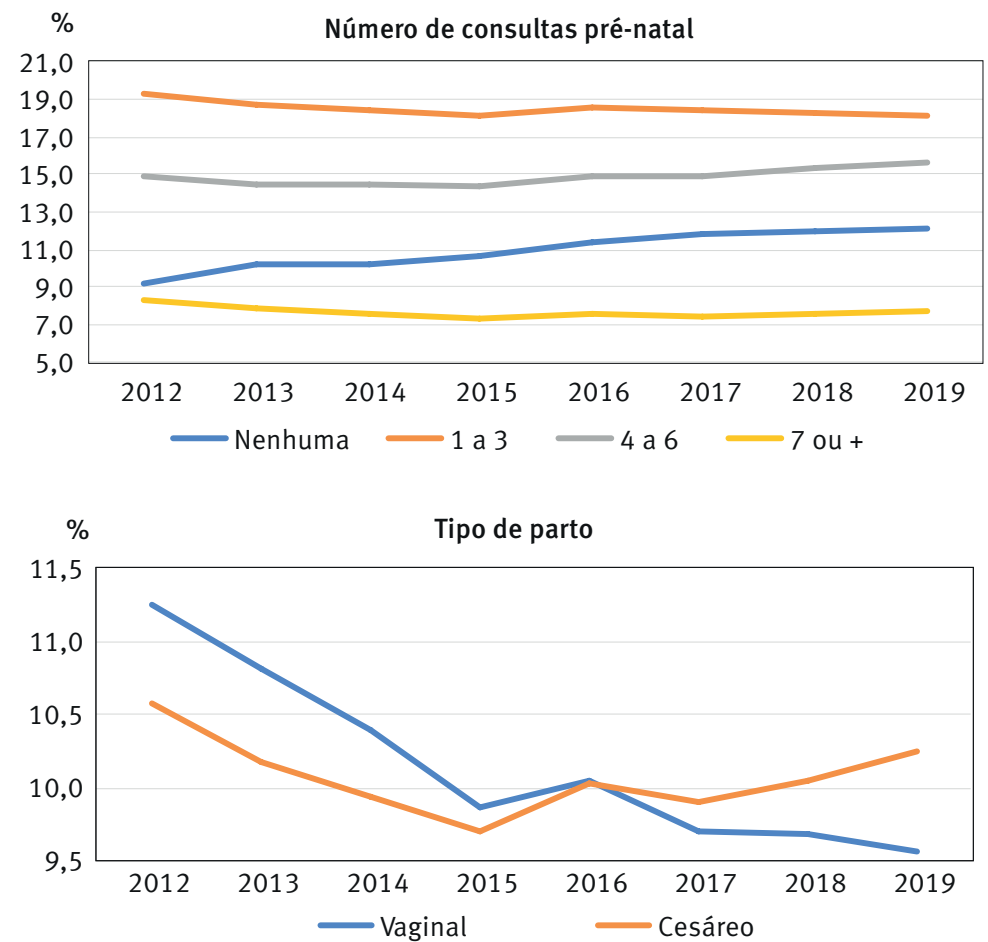

Fonte: Sistema de Informações sobre Nascidos Vivos (Sinasc). Elaboração dos autores.

Ao analisar a prematuridade segundo a idade materna, verifica-se que as mulheres na faixa etária de 10 a 14 anos apresentaram tendência decrescente de 2012 a 2015, crescente de 2016 a 2017 e decrescente a partir de 2018. Aquelas de 15 a 34 anos registraram movimento decrescente para todo o período, enquanto para as de 35 a 44 anos houve redução de 2012 a 2015 e aumento a partir de 2016. Já as mulheres com 45 anos ou mais mostraram tendência crescente para todo o período (Tabela 2). 
TABELA 2

Análise de tendência temporal da proporção de prematuridade, segundo idade materna, escolaridade, raça/cor, número de consultas pré-natal e tipo de parto

Brasil - 2012-2019

\begin{tabular}{|c|c|c|c|c|c|c|c|c|}
\hline Variáveis & Modelo & $\mathrm{R}$ & $\mathrm{R}^{2}$ & $\beta_{0}$ & $\beta_{1}$ & $\beta_{2}$ & $\beta_{3}$ & $\mathrm{p}$-valor \\
\hline \multicolumn{9}{|l|}{ Idade materna } \\
\hline \multirow[t]{3}{*}{ 10-14 anos } & Linear & 0,596 & 0,494 & 15,896 & $-0,071$ & - & - & 0,072 \\
\hline & Parábola & 0,759 & 0,598 & 15,822 & $-0,071$ & 0,025 & - & 0,118 \\
\hline & Terceiro grau & 0,984 & 0,961 & 15,822 & 0,044 & 0,025 & $-0,023$ & 0,023 \\
\hline 15-19 anos & Linear & 0,919 & 0,899 & 11,382 & $-0,185$ & - & - & 0,002 \\
\hline 20-34 anos & Linear & 0,727 & 0,659 & 9,312 & $-0,102$ & - & - & 0,031 \\
\hline \multirow[t]{2}{*}{ 35-39 anos } & Linear & 0,327 & 0,159 & 11,354 & $-0,065$ & - & - & 0,235 \\
\hline & Parábola & 0,957 & 0,928 & 11,175 & $-0,065$ & 0,061 & - & 0,009 \\
\hline \multirow[t]{2}{*}{ 40-44 anos } & Linear & 0,077 & $-0,154$ & 13,036 & $-0,031$ & - & - & 0,594 \\
\hline & Parábola & 0,980 & 0,967 & 12,827 & $-0,031$ & 0,072 & - & 0,003 \\
\hline 45 anos ou mais & Linear & 0,932 & 0,916 & 16,800 & 0,301 & - & - & 0,002 \\
\hline \multicolumn{9}{|l|}{ Escolaridade } \\
\hline Analfabeta & Linear & 0,730 & 0,663 & 12,327 & $-0,160$ & - & - & 0,030 \\
\hline 1-7 anos & Linear & 0,749 & 0,686 & 11,354 & $-0,098$ & - & - & 0,026 \\
\hline \multirow[t]{2}{*}{ 8-11 anos } & Linear & 0,533 & 0,416 & 9,686 & $-0,074$ & - & - & 0,099 \\
\hline & Parábola & 0,940 & 0,900 & 9,557 & $-0,074$ & 0,044 & - & 0,015 \\
\hline \multirow[t]{2}{*}{12 anos ou mais } & Linear & 0,521 & 0,402 & 9,686 & $-0,067$ & - & - & 0,105 \\
\hline & Parábola & 0,946 & 0,910 & 9,566 & $-0,067$ & 0,041 & - & 0,013 \\
\hline \multicolumn{9}{|l|}{ Raça/cor } \\
\hline \multirow[t]{2}{*}{ Branca } & Linear & 0,451 & 0,314 & 9,905 & $-0,076$ & - & - & 0,144 \\
\hline & Parábola & 0,920 & 0,866 & 9,749 & $-0,076$ & 0,053 & - & 0,023 \\
\hline Preta & Linear & 0,880 & 0,850 & 10,458 & $-0,188$ & - & - & 0,006 \\
\hline Amarela & Linear & 0,692 & 0,614 & 10,083 & $-0,085$ & - & - & 0,040 \\
\hline Parda & Linear & 0,801 & 0,752 & 10,028 & $-0,127$ & - & - & 0,016 \\
\hline Indígena & Linear & 0,975 & 0,969 & 13,887 & $-0,528$ & - & - & $<0,001$ \\
\hline \multicolumn{9}{|l|}{$\begin{array}{l}\text { Número de } \\
\text { consultas } \\
\text { pré-natal }\end{array}$} \\
\hline Nenhuma & Linear & 0,988 & 0,985 & 10,975 & 0,440 & - & - & $<0,001$ \\
\hline \multirow[t]{3}{*}{1 a 3} & Linear & 0,625 & 0,531 & 18,440 & $-0,081$ & - & - & 0,061 \\
\hline & Parábola & 0,802 & 0,670 & 18,354 & $-0,081$ & 0,029 & - & 0,088 \\
\hline & Terceiro grau & 0,994 & 0,985 & 18,354 & 0,037 & 0,029 & $-0,023$ & 0,009 \\
\hline 4 a 6 & Linear & 0,790 & 0,738 & 14,783 & 0,156 & - & - & 0,018 \\
\hline \multirow[t]{2}{*}{7 ou mais } & Linear & 0,399 & 0,249 & 7,589 & $-0,057$ & - & - & 0,179 \\
\hline & Parábola & 0,952 & 0,921 & 7,455 & $-0,057$ & 0,046 & - & 0,010 \\
\hline \multicolumn{9}{|l|}{ Tipo de parto } \\
\hline Vaginal & Linear & 0,925 & 0,906 & 10,102 & $-0,222$ & - & - & 0,002 \\
\hline \multirow[t]{2}{*}{ Cesário } & Linear & 0,081 & $-0,149$ & 9,998 & $-0,020$ & - & - & 0,585 \\
\hline & Parábola & 0,898 & 0,831 & 9,870 & $-0,020$ & 0,044 & - & 0,032 \\
\hline
\end{tabular}

Fonte: Sistema de Informações sobre Nascidos Vivos (Sinasc). Elaboração dos autores. 
As mulheres analfabetas ou com escolaridade de 1 a 7 anos apresentaram tendência decrescente na proporção de prematuridade para o período, enquanto aquelas com oito anos ou mais de estudo registraram redução de 2012 a 2015 e aumento a partir de 2016. Quanto à raça/cor, para as mulheres brancas a prematuridade diminuiu de 2012 a 2015 e cresceu a partir de 2016; já as mulheres pretas, amarelas, pardas e indígenas mostraram tendência decrescente para todo o período (Tabela 2).

Para as mulheres que não realizaram pré-natal ou tiveram de 4 a 6 consultas, observou-se elevação na proporção de prematuridade para o período, enquanto aquelas com 1 a 3 consultas registraram tendência decrescente de 2012 a 2015, crescente até 2016 e decrescente a partir de 2017. Para as mulheres com sete ou mais consultas verificaram-se redução da prematuridade de 2012 a 2015 e aumento a partir de 2016. No período estudado, a proporção de prematuridade diminuiu entre os partos vaginais, enquanto para os cesáreos a tendência foi decrescente de 2012 a 2015 e crescente a partir de 2016 (Tabela 2).

\section{Discussão}

De 2012 a 2019, a proporção de prematuridade no Brasil mostrou um comportamento de redução, variando de $10,87 \%$ a $9,95 \%$. As mulheres com idade materna avançada e com número de consultas pré-natal abaixo do recomendado pelo Ministério da Saúde apresentaram as maiores proporções de prematuridade para o período e com tendência crescente. Já as mulheres analfabetas e indígenas registraram tendência decrescente para o período, apesar de terem as maiores proporções de prematuridade.

O Brasil ainda apresenta uma proporção elevada de prematuridade em relação aos países europeus (8,7\%) (CHAWANPAIBOON et al., 2019), a despeito da tendência de queda para o período. Tal movimento é contrário ao que ocorreu no resto do mundo, em que a prematuridade passou de 9,8\% em 2000 para 10,6\% em 2014 (CHAWANPAIBOON et al., 2019). É provável que essa mudança deva-se ao novo modelo obstétrico adotado no Brasil: o Ministério da Saúde lançou diretrizes - Diretriz de Atenção à Gestante: a operação cesariana (BRASIL, 2015) e Diretriz Nacional de Assistência ao Parto Normal (BRASIL, 2017) - voltadas para as intervenções obstétricas, em especial a cesariana, principal causa de prematuridade por intervenção obstétrica no país (LEAL et al., 2016).

Essas diretrizes orientam as mulheres brasileiras, os profissionais de saúde e os gestores, tanto no âmbito público como no privado, sobre as situações relacionadas às vias de parto, suas indicações e condutas. Leal et al. (2019), ao compararem inquéritos realizados em 2012 e 2017 com puérperas de todo o Brasil, encontraram, para o setor privado, redução nas taxas de cesariana e aumento da idade gestacional. Tal fato mostra uma possível mudança no cenário do inquérito de 2012, com redução dos nascimentos prematuros em consequência de intervenção obstétrica (39,3\% do total de prematuros), dos quais mais de $90 \%$ ocorreram via cesariana eletiva, especialmente prematuros tardios (LEAL et al., 2016). 
Outro ponto a ser destacado é a tendência decrescente da falta de preenchimento sobre semana gestacional do recém-nascido no Sinasc. Estudo realizado com dados do Sistema de Informações sobre Mortalidade (SIM) e Sinasc, nos triênios 1999-2001 e 2008-2010, reconhece que a cobertura e a qualidade dos dados vêm melhorando acentuadamente, alcançando índices de adequação de 89,1\% e 90,9\%, respectivamente (FRIAS; SZWARCWALD; LIRA, 2014).

A literatura mostra que as maiores proporções de prematuridade ocorrem em mulheres mais vulneráveis, com pouca escolaridade, pré-natal inadequado, raça/cor não branca, em extremos etários (DONGARWAR et al., 2021; OLIVEIRA et al., 2019). Isso corrobora nossas análises, entretanto, é importante destacar que os serviços de saúde têm conseguido minimizar, em parte, o efeito desses fatores de vulnerabilidade, já que mulheres com nenhuma ou pouca escolaridade, pretas, amarelas, pardas e indígenas registraram tendência decrescente para todo o período.

Contudo, os serviços de saúde ainda apresentam falhas na atenção pré-natal, uma vez que a inadequação do pré-natal mostrou tendência crescente na proporção de prematuridade durante todo o período. A maioria das mulheres realiza pré-natal, entretanto, a qualidade da assistência não é satisfatória, pois apenas 15\% das 6.125 mulheres que realizaram pré-natal nas unidades básicas de saúde do Brasil, em 2012-2013, receberam todos os procedimentos, exames e orientações preconizados pelo Ministério da Saúde (TOMASI et al., 2017). Assim, a identificação do risco gestacional pelo profissional de saúde e o encaminhamento em tempo oportuno, quando necessário, ficam comprometidos, levando a desfechos neonatais desfavoráveis, como a prematuridade (OLIVEIRA et al., 2019).

No que concerne à idade da mãe, as mulheres na faixa etária adequada para reprodução (20 a 34 anos) apresentaram tendência decrescente de prematuridade no período estudado, provavelmente devido à diminuição das intervenções obstétricas desnecessárias (LEAL et al., 2019). Já as mulheres em idade materna avançada ( 45 anos ou mais) registraram tendência crescente, possivelmente em função do aumento do número de reproduções assistidas no Brasil, que, por sua vez, aumentam a possibilidade de gestações múltiplas, as quais representam fator de risco para o parto pré-termo (BITTAR; ZUGAIB, 2009; LAKRYC et al., 2019). Além disso, essas mulheres, principalmente nulíparas, e seus obstetras possuem percepção de risco com um limiar mais baixo, podendo causar intervenções iatrogênicas (CLARAMONTE NIETO et al., 2019; MARIANI; VAIN, 2019; RYDAHL et al., 2019).

A proporção de prematuridade por parto vaginal apresentou tendência decrescente, mostrando que a prematuridade espontânea tem diminuído, provavelmente porque os profissionais de saúde têm identificado mulheres com trabalho de parto prematuro suspeito, diagnosticado ou estabelecido e feito intervenções com progesterona vaginal ou cerclagem uterina profiláticas quando indicado (NATIONAL COLLABORATING CENTRE FOR WOMEN'S AND CHILDREN'S HEALTH, 2015), postergando assim o nascimento e prevenindo desfechos negativos no recém-nascido. 
Entretanto, a proporção de prematuridade por parto cesáreo mostrou-se crescente no segundo período, apesar de as cesarianas eletivas terem diminuído entre 2012 e 2017 (LEAL et al., 2019). Isso pode ter acontecido porque as mulheres com complicações obstétricas continuam a precisar desse tipo de parto e, também, é possível que em algumas regiões do Brasil (Norte, Sudeste e Centro-Oeste) o número de cesarianas eletivas não tenha diminuído significativamente, já que a prematuridade moderada a tardia mostrou-se crescente no segundo período.

Este trabalho destaca-se por apresentar uma análise temporal da prematuridade no Brasil após a mudança na forma de registro da duração da gestação com dados oficiais, antes subestimada quando comparada aos estudos com dados primários (MATIJASEVICH et al., 2013). Outro ponto positivo é que o Sinasc possui alta cobertura e adequação dos dados, com valores próximos a 90\% (FRIAS; SZWARCWALD; LIRA, 2014). Além disso, a análise de tendência ocorreu segundo subcategorias da prematuridade, regiões do Brasil, idade materna, escolaridade materna, raça/cor, número de consultas pré-natal e tipo de parto. Entretanto, possui limitações inerentes a qualquer estudo que envolve dados secundários, como confiabilidade dos dados e não inferência em nível individual.

Apesar de a proporção de prematuridade ainda ser elevada, ao contrário do resto do mundo, o Brasil tem mostrado uma tendência decrescente. Ela ocorre principalmente em mulheres mais vulneráveis, com baixa escolaridade e não brancas. Entretanto, para as mulheres com idade materna avançada e com poucas consultas de pré-natal, os serviços de saúde ainda têm falhado na identificação do risco gestacional e encaminhamento em tempo oportuno para que esse desfecho seja evitado.

\section{Referências}

ABDEL RAZEQ, N. M.; KHADER, Y. S.; BATIEHA, A. M. The incidence, risk factors, and mortality of preterm neonates: a prospective study from Jordan (2012-2013). Journal of Turkish Society of Obstetric and Gynecology, v. 14, p. 28-36, Mar. 2017.

BALBI, B.; CARVALHAES, M. A. de B. L.; PARADA, C. M. G. de L. Tendência temporal do nascimento pré-termo e de seus determinantes em uma década. Ciência \& Saúde Coletiva, v. 21, n. 1, p. 233-241, jan. 2016.

BITTAR, R. E.; ZUGAIB, M. Indicadores de risco para o parto prematuro: [revisão]. Revista Brasileira de Ginecologia e Obstetrícia, v. 31, n. 4, p. 203-209, abr. 2009.

BRASIL. Ministério da Saúde. Manual de instruções para o preenchimento da Declaração de Nascido Vivo. Brasília: Departamento de Análise da Situação de Saúde, Secretaria de Vigilância em Saúde, 2011.

BRASIL. Ministério da Saúde. Diretrizes de Atenção à Gestante: a operação cesariana. Brasília: Conselho Nacional de Incorporação de Tecnologias no SUS, 2015.

BRASIL. Ministério da Saúde. Diretrizes Nacionais de Assistência ao Parto Normal. Brasília: Conselho Nacional de Incorporação de Tecnologias no SUS, 2017.

CHAWANPAIBOON, S. et al. Global, regional, and national estimates of levels of preterm birth in 2014: a systematic review and modelling analysis. The Lancet Global Health, v. 7, n. 1, p. e37-e46, Jan. 2019. 
CLARAMONTE NIETO, M. et al. Impact of aging on obstetric outcomes: defining advanced maternal age in Barcelona. BMC Pregnancy and Childbirth, v. 19, n. 1, Article 342, Dez. 2019.

DONGARWAR, D. et al. Temporal trends in preterm birth phenotypes by plurality: black-white disparity over half a century. Journal of Perinatology, v. 41, n. 2, p. 204-211, Feb. 2021.

FRIAS, P. G. de; SZWARCWALD, C. L.; LIRA, P. I. C. de. Avaliação dos sistemas de informações sobre nascidos vivos e óbitos no Brasil na década de 2000. Cadernos de Saúde Pública, v. 30, n. 10, p. 2068-2280, out. 2014.

GONZAGA, I. C. A. et al. Atenção pré-natal e fatores de risco associados à prematuridade e baixo peso ao nascer em capital do Nordeste brasileiro. Ciência \& Saúde Coletiva, v. 21, n. 6, p. 1965-1974, jun. 2016.

HOWSON, C. P.; KINNEY, M. V.; LAWN, J. E. (ed.). Born too soon: the global action report on preterm birth. Geneve: World Health Organization, 2012. Disponível em: http://www.who.int/ pmnch/media/news/2012/201204\%5Fborntoosoon-report.pdf.

HUANG, H. et al. Investigation of association between environmental and socioeconomic factors and preterm birth in California. Environment International, v. 121, p. 1066-1078, Dec. 2018.

LAKRYC, E. et al. Modelo de impacto orçamentário da aplicação do single embryo transfer consecutivo comparado com o double embryo transfer em tratamentos de fertilização in vitro no Sistema de Saúde Suplementar. Jornal Brasileiro de Economia da Saúde, v. 11, n. 3, p. 231243, dez. 2019.

LATORRE, M. do R. D. de O.; CARDOSO, M. R. A. Análise de séries temporais em epidemiologia: uma introdução sobre os aspectos metodológicos. Revista Brasileira de Epidemiologia, v. 4, n. 3, p. 145-152, nov. 2001.

LEAL, M. do C. et al. Prevalence and risk factors related to preterm birth in Brazil. Reproductive Health, v. 13, suppl. 3, p. 163-174, Oct. 2016.

LEAL, M. do C. et al. Avanços na assistência ao parto no Brasil: resultados preliminares de dois estudos avaliativos. Cadernos de Saúde Pública, v. 35, n. 7, e00223018, 2019.

MARIANI, G. L.; VAIN, N. E. The rising incidence and impact of non-medically indicated pre-labour cesarean section in Latin America. Seminars in Fetal and Neonatal Medicine, v. 24, n. 1, p. 11-17, Feb. 2019.

MARTIN, J. A. et al. Births in the United States, 2016. National Center for Health Statistics, 2017. (NCHS Data Brief, n. 287).

MATIJASEVICH, A. et al. Estimativas corrigidas da prevalência de nascimentos pré-termo no Brasil, 2000 a 2011. Epidemiologia e Serviços de Saúde, v. 22, n. 4, p. 557-564, dez. 2013.

NATIONAL COLLABORATING CENTRE FOR WOMEN'S AND CHILDREN'S HEALTH (UK). Preterm labour and birth. London, UK: National Institute for Health and Care Excellence (UK), 2015.

OLIVEIRA, A. A. de et al. Fatores associados ao nascimento pré-termo: da regressão logística à modelagem com equações estruturais. Cadernos de Saúde Pública, v. 35, n. 1, e00211917, 2019.

RYDAHL, E. et al. Cesarean section on a rise - Does advanced maternal age explain the increase? A population register-based study. PLOS ONE, v. 14, n. 1, e0210655, 24 Jan. 2019.

SILVEIRA, M. F. et al. Nascimentos pré-termo no Brasil entre 1994 e 2005 conforme o Sistema de Informações sobre Nascidos Vivos (Sinasc). Cadernos de Saúde Pública, v. 25, n. 6, p. 12671275, jun. 2009.

TOMASI, E. et al. Qualidade da atenção pré-natal na rede básica de saúde do Brasil: indicadores e desigualdades sociais. Cadernos de Saúde Pública, v. 33, n. 3, 2017. 


\title{
Sobre os autores
}

Katrini Guidolini Martinelli é cirurgiã-dentista, doutora em epidemiologia em saúde pública pela Escola Nacional de Saúde Pública Sérgio Arouca - Fiocruz. Pós-doutoranda do Programa de Pós-graduação em Saúde Coletiva da Universidade Federal do Espírito Santo.

Barbara Almeida Soares Dias é enfermeira, doutora em epidemiologia em saúde pública pela Escola Nacional de Saúde Pública Sérgio Arouca - Fiocruz.

Marcelle Lemos Leal é bióloga, doutora em saúde coletiva pela Universidade Federal do Espírito Santo. Coordenadora da vigilância epidemiológica na Prefeitura de Marataízes-ES.

Lorrayne Belotti é cirurgiã-dentista, doutoranda em saúde pública na Universidade de São Paulo e mestra em saúde coletiva pela Universidade Federal do Espírito Santo.

Érica Marvila Garcia é enfermeira, doutoranda em saúde pública na Universidade de São Paulo e mestra em saúde coletiva pela Universidade Federal do Espírito Santo.

Edson Theodoro dos Santos Neto é cirurgião-dentista, doutor em epidemiologia em saúde pública pela Escola Nacional de Saúde Pública Sérgio Arouca - Fiocruz. Professor associado do Departamento de Medicina Social da Universidade Federal do Espírito Santo.

\section{Endereço para correspondência}

\author{
Katrini Guidolini Martinelli \\ Programa de Pós-graduação em Saúde Coletiva \\ Av. Marechal Campos, 1468, Maruípe \\ 29040-090 - Vitória-ES, Brasil \\ Barbara Almeida Soares Dias \\ Programa de Pós-graduação em Saúde Coletiva \\ Av. Marechal Campos, 1468, Maruípe \\ 29040-090 - Vitória-ES, Brasil \\ Marcelle Lemos Leal \\ Programa de Pós-graduação em Saúde Coletiva \\ Av. Marechal Campos, 1468, Maruípe \\ 29040-090 - Vitória-ES, Brasil \\ Lorrayne Belotti \\ Departamento de Política, Gestão e Saúde \\ Av. Dr. Arnaldo, 715, Cerqueira César \\ 01246-904 - São Paulo-SP, Brasil \\ Érica Marvila Garcia \\ Departamento de Epidemiologia \\ Av. Dr. Arnaldo, 715, Cerqueira César \\ 01246-904 - São Paulo-SP, Brasil \\ Edson Theodoro dos Santos Neto \\ Departamento de Medicina Social \\ Av. Marechal Campos, 1468, Maruípe \\ 29040-090 - Vitória-ES, Brasil
}




\begin{abstract}
Preterm births in Brazil between 2012 and 2019: data from the Information System on Live Births
\end{abstract}

The aim of this study is to analyze the trend of prematurity in Brazil from 2012 to 2019, according to sociodemographic, prenatal and childbirth characteristics. This is an ecological time series study, in which the proportions of prematurity were calculated according to the regions of Brazil, maternal age, maternal education, race/color, number of prenatal consultations and type of delivery from the data of Information System on Live Births (SINASC). Sequentially, polynomial regression models were applied to analyze time trends. From 2012 to 2019, the proportion of prematurity in Brazil showed a decreasing trend, ranging from $10.87 \%$ to $9.95 \%$, with the lowest proportion in 2015, which was $9.77 \%$. Women aged $\geq 45$ years and with 4 to 6 prenatal consultations had the highest proportions of prematurity for the period (14.88\% to $17.92 \%)$ and with an increasing trend. Illiterate and indigenous women, on the other hand, showed a decreasing trend for the period, despite having the highest proportions of prematurity $(15.75 \%$ to $11.74 \%$ ). There is a decreasing trend of prematurity in Brazil, especially in more vulnerable women. However, health services need to improve the care of women with an advanced maternal age and attract women with few prenatal consultations.

Keywords: Infant premature. Premature birth. Information systems. Health planning.

\title{
Resumen
}

Prematuridad en Brasil entre 2012 y 2019: datos del Sistema de Información de Nacidos Vivos

El objetivo de este estudio es analizar la tendencia de la prematuridad en Brasil entre 2012 y 2019, según características sociodemográficas, prenatales y del parto. Se trata de un estudio ecológico de series temporales, en el que se calcularon las proporciones de prematuridad según las regiones de Brasil, la edad materna, la educación materna, la adscripción de etniaraza, el número de consultas prenatales y el tipo de parto, a partir de los datos del Sistema de Información sobre Nacidos Vivos (SINASC). Para ello se aplicaron secuencialmente modelos de regresión polinomial para analizar las tendencias temporales. Desde 2012 hasta 2019, la proporción de prematuridad en Brasil mostró una tendencia decreciente, que va del 10,87 \% al $9,95 \%$, con la proporción más baja en 2015 , que fue de $9,77 \%$. Las mujeres de 45 años o más y con cuatro a seis consultas prenatales tuvieron las mayores proporciones de prematurez del período (14,88 \% a 17,92 \%) y con una tendencia creciente. Las mujeres analfabetas e indígenas, por su parte, mostraron una tendencia decreciente para el período, a pesar de tener las mayores proporciones de prematuridad (entre $15,75 \%$ y $11,74 \%$ ). Existe una tendencia decreciente de la prematuridad en Brasil, especialmente entre las mujeres más vulnerables a pesar de lo cual los servicios de salud deben mejorar la atención de las mujeres con edad materna avanzada y atraer a mujeres con pocas consultas prenatales.

Palabras clave: Recién nacido prematuro. Nacimiento prematuro. Sistemas de información. Planificación en salud. 